\title{
Hybrid Gain Medium used in Multiwavelength Ring Laser for Noise Reduction
}

\author{
K.Suganya \\ Assistant Professor \\ I.F.E.T College of Engineering \\ Villupuram, \\ Tamilnadu, India
}

\author{
D.Shanmuga Sundar \\ Research Scholar \\ Alagappa Chettiar College of Engineering and \\ Technology \\ Karaikudi, Tamilnadu, India
}

\begin{abstract}
Multiwavelength ring laser based on hybrid gain medium with semiconductor optical amplifier and erbium doped fiber amplifier plays a vital role in noise reduction. The proposed scheme is evaluated with output power, long term stability and side mode suppression using hybrid gain medium. More than 40 lines with high output power is achieved in this set up. The wavelength interval between the channels is $0.29 \mathrm{~nm}$. The laser can be tuned from $1545 \mathrm{~nm}$ to $1555 \mathrm{~nm}$. The multiwavelength ring laser with $0 \mathrm{~dB}$ noise power is used for broad band transmission and various applications.
\end{abstract}

\section{Keywords}

Multiwavelength Ring Laser, Delay interferometer, EDFA, SOA.

\section{INTRODUCTION}

Multiwavelength laser source have potential applications in optical fiber sensors, optical signal processing, sensor network multiplexing scheme and instrument testing. Multiwavelength simultaneously emits different wavelength channel which provide an efficient way to increase the transmission capability of WDM (Wavelength Division Multiplexing) system, since the number of wavelength generated is proportional to the system transmission capacity and system reliability. They also have a variety of advantages, such as multiwavelength operation, simple structure, low cost, narrow line width, high power, low intensity noise and compatibility with other optical fiber components [3].

Multiwavelength laser operation is achieved by various gain mechanisms, which includes erbium doped fiber (EDF) amplification, semiconductor optical amplification and fiber Raman amplification [4]. EDFA consist of a length of silica fiber whose core is doped with an ionized atom, Er3+ (Erbium). EDFA is used to drive multiwavelength laser source due to its lower threshold and high power conversion efficiency. However, since erbium doped fiber (EDF) is a homogenous gain medium, the laser using EDF as a gain mechanism suffers from strong mode competition and unstable multi-wavelength lasing at room temperature. This also leads to power fluctuation. There have been a range of approaches used to solve this problem which includes cooling EDF to liquid-nitrogen temperature, using inhomogeneous gain medium in the laser cavity such as semiconductor optical amplifier (SOA) [1], [2] instead of EDF, utilizing four wave mixing effect [3] or inhomogeneous loss mechanism by using highly-nonlinear fiber, and adding a frequency shifter or phase modulator and so on. SOA-based laser can be an alternative for multiwavelength DWDM transmitters due to its broad gain bandwidth and stable wavelength spacing at room temperature. However, the SOA gain spectrum is usually parabolic and not flat within the operation wavelength range.
Hence, it needs to be flattened to achieve multiwavelength lasing with identical channel separation and output power [7]. SOA has many advantages: it has good short-term stability, it can generate multiwavelength oscillation at room temperature, and it can be directly mode locked at high bit-rate by optical injection. The intrachannel mode competition at each wavelength channel is resolved by using a semiconductor optical amplifier as a gain medium.

In reference [5], it is reported a novel multiwavelength SOAbased ring laser utilizing the Vernier effect to provide fine selection of lasing modes and improved spectral noise performance. However they utilized a coupled dual cavity, in which the ratio of the length of two rings should be maintained for the side mode suppression.

In this paper, the side mode suppression and stable multiwavelength is achieved by hybrid gain medium (EDFA and SOA) in the ring cavity. Simultaneous generation of 40 wavelength channel is achieved with high output power. This multiwavelength ring laser can be used for broad band transmission over $12 \mathrm{kM}$ standard single mode fiber at a channel data rate of $10 \mathrm{~Gb} / \mathrm{s}$.

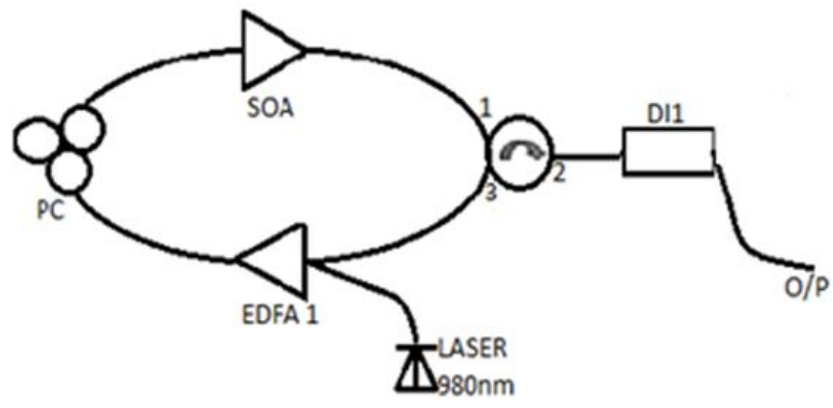

Fig 1: Pictorial representation of multiwavelength ring laser

\section{OPERATING PRINCIPLE}

The setup consists of ring structure as shown in the fig. 1. It consists of an SOA, an EDFA, polarization controller, circulator and delay interferometer. EDFA and SOA act as the gain medium. An EDFA with $5 \mathrm{~m}$ length erbium doped fiber is used. It is driven by a continuous wave laser at a center frequency of $1550 \mathrm{~nm}$. The input power is set at $27 \mathrm{dbm}$. The SOA with broad gain spectrum is used in the cavity for side mode suppression. It is also used to suppress the noise that is generated due to continuous oscillation The circulator used here is a three port circulator, an input signal on port1 is sent out on port 2 , an input signal on port 2 is sent out on port 3 and an input signal on port3 is sent out on port1. Here the circulator transfers the output from ring cavity to the delay 
interferometer and from delay interferometer to the ring cavity. A polarization controller was employed to control the polarization state in the laser cavity. The injection current is set at $0.3 \mathrm{~A}$. The single longitudinal mode (SLM) operation is achieved by using $5 \mathrm{~mm}$ long SOA.

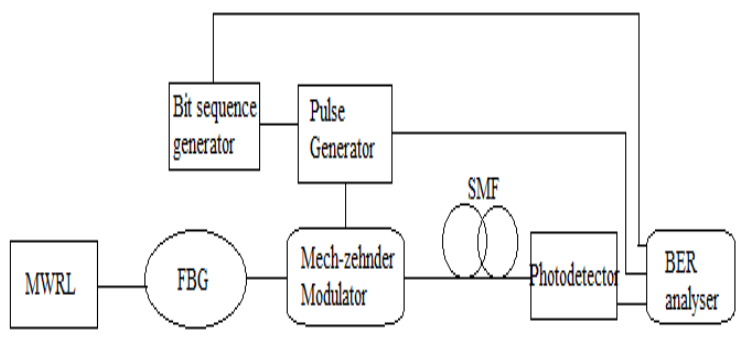

Fig 2: Schematic set up of MWRL for broad band transmission: FBG, fiber Bragg grating; SMF, single mode fiber.

The DI which is fabricated using $\mathrm{Si}-\mathrm{SiO} 2$ waveguide technology has two arms with a 25 ps time delay between the two arms [6]. The light is split into two beams, and, at the output of DI, two beams of optical signals with the phase difference (corresponding to $25 \mathrm{ps}$ delay) recombine and interfere with each other.

The optical intensity at the DI output can be given

$$
\text { Iout }(\lambda)=\operatorname{Iup}(\lambda)+\operatorname{Ilow}(\lambda)+2 \sqrt{ } \operatorname{Iup}(\lambda) * \operatorname{Ilow}(\lambda) \cos \Delta \theta
$$

\section{$\Delta \theta=2 \Pi C^{*} \Delta t / \lambda$}

where Iup and Ilow are intensity of two beams travelling along the two arms of the DI, $t$ is the travel time delay between the two arms; $c$ is the speed of light in vacuum and $\lambda$ is the wavelength of the optical signal. The DI acts as a comb filter which makes the wavelengths that match the peaks of the comb oscillate when it is in a laser cavity. The wavelength spacing between two adjacent transmission peaks is $0.32 \mathrm{~nm}$ for $t=25 \mathrm{ps}$. The line width of the peaks is reduced for the two trip transmission (equivalent to two cascaded DIs). Multiple transmissions through identical DIs will further reduce the line width of the transmission peaks. By increasing the time delay of the delay interferometer the wavelength spacing is reduced and increase the number of wavelength channel. The light is split into two beams and one of the outputs is given back to the circulator and back to the EDFA.

In the ring laser the longitudinal mode spacing $\Delta \mathrm{v}$ is given by

$$
\Delta \mathrm{v}=\mathrm{c} / \mathrm{nL}
$$

Where $\mathrm{c}$ is the speed of the light in vacuum, $\mathrm{n}$ is the refractive index of the fiber core and $\mathrm{L}$ is the length of the ring cavity.

Fig. 2 shows the schematic set up of MWRL for broad band transmission. Numerous wavelength channel are emitted by MWRL and a single wavelength is selected by fiber bragg grating (FBG). To obtain a particular wavelength, the refractive index and center frequency is set appropriately. The FBG should have $99 \%$ reflectivity with center frequency $1550 \mathrm{~nm}$. The bandwidth and the dispersion of FBG are $40 \mathrm{GHz}$ and $-800 \mathrm{ps} / \mathrm{nm}$ respectively. The tuned wavelength is modulated using mech-zehnder modulator with pulse generator and bit sequence generator. The modulated signal is transmitted through single mode fiber of desired length and it is detected by any photodetector. The output single is analyzed with BER analyzer.

\section{EXPERIMENTAL RESULT AND ANALYSIS}

Fig. 3 shows the implemented circuit in the Optisystem software. Optisystem is a mature software package provides comparable simulation results.

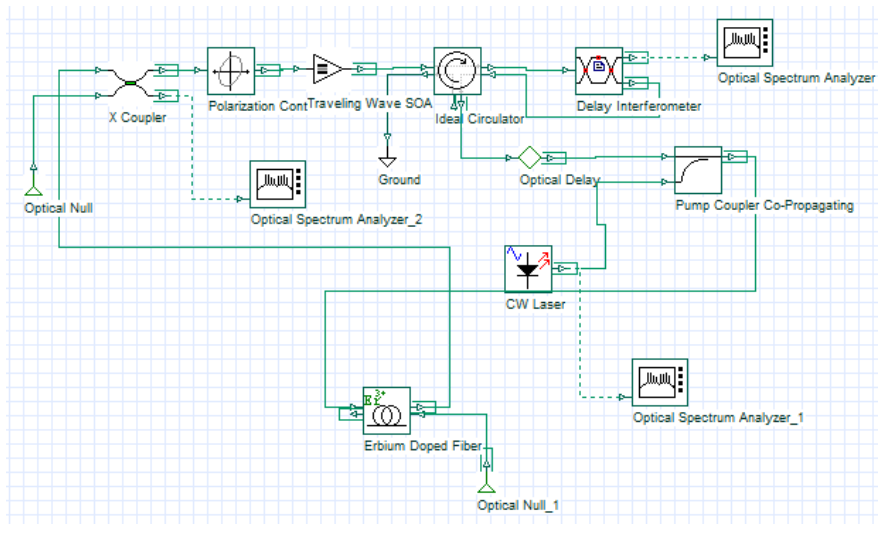

Fig 3: Implemented circuit in Optisystem

The erbium doped fiber with appropriate length of $5 \mathrm{~m}$ and the numerical aperture 0.24 was designed. The core radius of EDFA is 2.2um. The pump coupler couples the feedback output with an optical delay and an input from continuous wave laser. The centre frequency of the continuous wave laser is set at $1550 \mathrm{~nm}$ with $27 \mathrm{dBm}$ output power. The line width is set at $10 \mathrm{MHz}$.The azimuth and ellipticity angle of the polarization controller is calculated by using

$$
\mathrm{E}=45^{\circ}-\beta
$$

Where $\mathrm{E}$ is an ellipticity angle and $\beta$ is an azimuth angle. The layout parameter are set with the value as sequence length 128 bits, samples per bit 64 and number of samples 8192 . Coupling coefficient of the coupler is set as 0.1 . That is only $10 \%$ of the output is given to the polarization controller. The well packaged DI with the time delay of $25 \mathrm{ps}$ is important in eliminating the influence of thermal and power fluctuation in the ring.

\section{RESULT AND DISCUSSIONS}

In the experiment, the ring structure was built to generate multiwavelength output. The current driven by SOA is $0.3 \mathrm{~A}$ and the feedback is given back to the EDFA through circulator. Fig. 4 (a) shows the single input wavelength which drives the EDFA with the center frequency $1550 \mathrm{~nm}$. At relaxation oscillation the single wavelength is converted into multiwavelength.

Fig. 4 (b) shows the noise spectrum generated in the ring cavity. The plot was obtained by using a high resolution optical spectrum analyzer which measure the optical spectra with a high resolution of $0.01 \mathrm{~nm}$. 


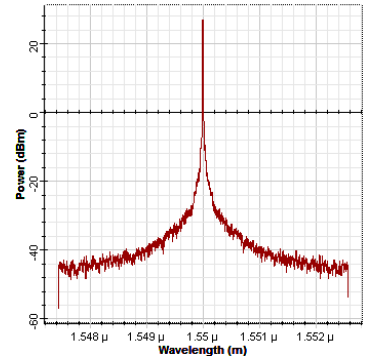

(a)

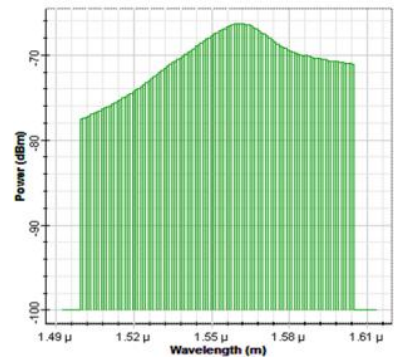

(b)
Fig 4: (a) Input spectrum and (b) Noise spectrum

The spectrums with green color represent the noise due to intra-channel mode competition at each wavelength channel. The coupling co-efficient of coupler was set as 0.1 ; in such a way that only $10 \%$ of the output is feed into the network. The noise was due to the Erbium dobed fiber amplifier in the ring cavity. The output from the coupler is fed back to the polarization controller which controls the polarization state in the cavity. Polarization controller is followed by SOA which is used to suppress the noise and intrachannel mode competition that is generated by EDFA. The circulator that circulates the output from SOA to the delay interferometer. The delay interferometer produces the multiwavelength output with noise power of $0 \mathrm{~W}$.

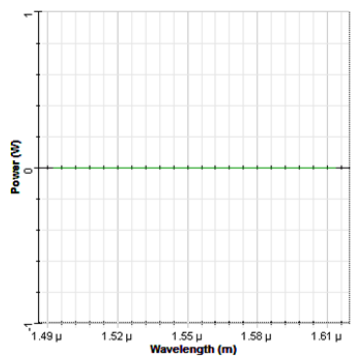

(a)

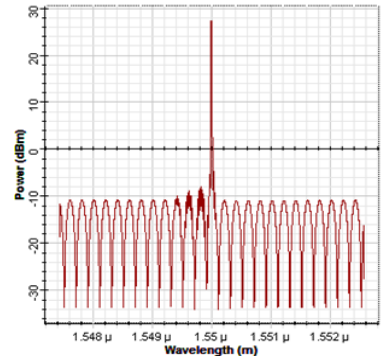

(b)
Fig 5: (a) noise reduction output from DI, (b) Multiwavelength output

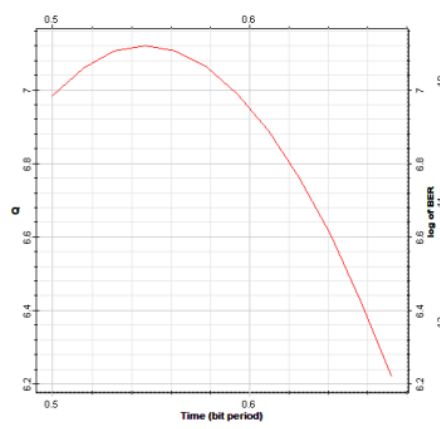

(a)

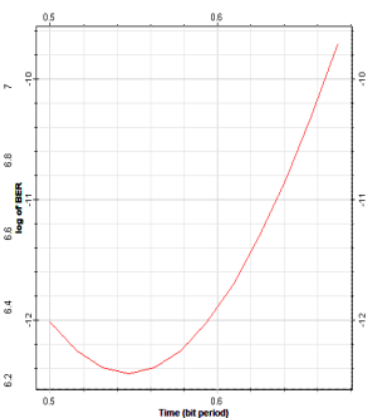

(b)
Fig 6: (a) $Q$ factor, (b) Min BER

Figure 5(a). Shows the noise reduction output from the delay interferometer. The noise was completed suppressed. Fig. 5(b) shows the multiwavelength output with number of wavelength channel. By increasing the delay interferometer the number of wavelength channel is increased. By using the optical tunable filter single wavelength can be selected. The hybrid gain medium effectively improves the SNR and supports stable multiwavelength lasing operation, and, the ring structure reshapes the transmission spectrum and generates lasing lines with narrow line width.

Fig. 6(a) \& (b) shows the Q factor and Min BER graph that is obtained after the broad band transmission. The Q factor is obtained as 7.1209 with min BER as 3.59244e-13.

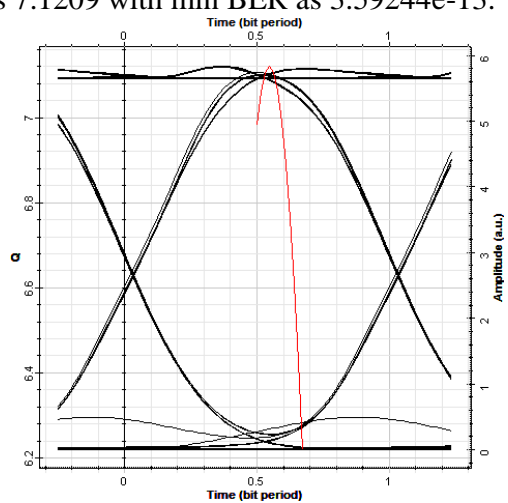

Fig 7: Eye opening with $Q$ factor

Fig. 7 shows the eye opening of the transmitted signal over $12 \mathrm{kM}$ a standard single mode fiber at a channel data rate of $10 \mathrm{~Gb} / \mathrm{s}$.

Table I shows the performance analysis of the output over a broad band transmission.

Table 1: Performance analysis

\begin{tabular}{|c|c|}
\hline Max.Q Factor & 7.1209 \\
\hline Min.BER & $3.59 \mathrm{E}-13$ \\
\hline Eye Height & 3.16573 \\
\hline Threshold & 0.710945 \\
\hline Decision Inst & 0.546875 \\
\hline
\end{tabular}

The analysis is made with single SOA in the cavity that produce lasing of 15 wavelength. The analysis is also made with double SOA, the lasing wavelength is increased upto 21 but the signal to noise ratio is reduced with the increase in side modes. Using the hybrid gain medium in the loop, lasing of 40 stable wavelengths is obtained with complete side mode suppression.

\section{CONCLUSION}

This paper presents a stable multiwavelength ring laser based on hybrid gain mediums, with a semiconductor optical amplifier and Erbium dobed fiber amplifier. The laser output is very stable with the complete reduction of noise level. The multiwavelength operation with the simultaneous lasing of more than 40 wavelengths with the frequency spacing of $50 \mathrm{GHz}$ was obtained. The side modes are suppressed into 0 level and a complete stable multiwavelength is obtained at the output. The obtained wavelength is also used for broad band transmission. The maximum Q factor is obtained as 7 with the minimum bit error rate. Further multiwavelength can be increased with the incorporation of several SOA and EDFA into the ring cavity.

\section{REFERENCES}

[1] QianfanXu and MinyuYao "Theoretical Analyses on Short-Term Stability of Semiconductor Fiber Ring Lasers"IEEE Journal of Quantum Electronics, Vol. 39, No.10 Oct 2003. 
[2] N.Pleros, T.Houbavlis, G.Theophilopoulos, K.Vlachos, C.Bintjas and H.Avramopoulos "SOA- Based Multi Wavelength Laser Sources" Photonics Communication Research Laboratory, 30 March 2004.

[3] Xiaochuan $\mathrm{Xu}$, Yong Yao and Deying Chen “ Numerical analysis of multiwavelength erbium -doped fiber ring laser exploiting four-wave mixing" Optics Express Vol. 16, No. 16 Aug 2008.

[4] Montserrat Fernandez-Vallejo, Silvia Diaz, Rosa AnaPerez Herrera, and Ruth Unzu, "Comparison of the Stability of Ring Resonator Structures for Multiwavelength Fiber Lasers Using Raman or Er-Doped Fiber Amplification" IEEE Journal of Quantum Electronics, Vol.45, No.12, December 2009.
[5] KwanilLee, SunDoLim, ChulHanKim, JuHanLee, Young-GeunHan and SangBaeLee "Noise Reduction in Multiwavelength SOA-Based Ring Laser by Coupled Dual Cavities for WDM Applications" Journal of lightwave Technology, Vol.28, No.5, March1, 2010

[6] Z. Chen, S. Ma and N. K. Dutta "Multiwavelength fiber ring laser based on a semiconductor and fiber gain medium" OPTICS EXPRESS, Vol. 17, No. 32 February 2009

[7] Junqiang Sun, Ying Zhang, and Xinliang Zhang "Multiwavelength Lasers Based on Semiconductor Optical Amplifiers" IEEE Photonics Technology letters, Vol.14, No.5, May 2002 